\title{
Introduction to drug allergy, and whom to refer for specialist assessment?
}

\author{
Authors: Bogusia Kasternow ${ }^{A}$ and Mohammed Yousuf Karim ${ }^{B}$
}

Drug allergy is defined as an adverse drug reaction with an established immunological mechanism. The National Institute for Health and Care Excellence published clinical guidelines on drug allergy in 2014 and quality standards in 2015. The intention of this article is to highlight indications for referral to specialists for management of drug allergy. Accurate diagnosis of drug allergy is critical of course for patient safety, but also for better use of the drugs that we have available and to reduce unnecessary avoidance of drugs. However, there are significant limitations in terms of resource availability and also in terms of testing. There is a careful balance here in that drug allergy is very common and clearly there is neither indication nor sufficient resource in the NHS for all patients with drug allergy to be reviewed by a specialist. It is, therefore, important to highlight to general physicians and physicians of other specialties, those patients who do require referral for specialist review.

\section{Introduction}

An adverse drug reaction (ADR) is defined as 'an appreciably harmful or unpleasant reaction resulting from an intervention related to the use of a medicinal product, which predicts hazard from future administration and warrants prevention or specific treatment, or alteration of the dosage regimen, or withdrawal of the product'. ${ }^{1}$ However, drug allergy has a more specific definition. It has been defined by the British Society for Allergy and Clinical Immunology (BSACI) as 'an ADR with an established immunological mechanism'. ${ }^{2,3}$ The subject of drug allergy is very topical, particularly with the focus on patient safety, and has attracted the attention of the National Institute for Health and Care Excellence (NICE) with publication of clinical guidelines on drug allergy in 2014 and quality standards in 2015., The intention of this article is not to provide a comprehensive review of drug allergy, but rather to introduce the topic and highlight indications for referral to specialists for management of drug allergy. Accurate diagnosis of drug allergy is critical for patient safety, for better use of the drugs that we have available and to reduce the unnecessary

Authors: A consultant allergist, Royal Surrey County Hospital, Surrey,

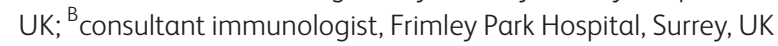

avoidance of drugs. However, there are significant limitations in terms of resource availability and also in terms of testing. There is a careful balance here in that drug allergy is very common and clearly there is neither indication nor sufficient resource in the NHS for all patients with drug allergy to be reviewed by a specialist.

\section{Assessment}

There is an issue of perception versus reality with respect to drug allergy. The prevalence of drug allergy estimated by patients in the general population is much higher than can be found objectively. For example, there are significant differences in the perception of the frequency of penicillin allergy. ${ }^{5}$ This raises an important point in the initial evaluation of the patient with suspected drug allergy. In most cases, clinical assessment will establish the diagnosis of patients with drug allergy, as opposed to other ADRs, and also help to establish the nature of the reaction. The assessment based on the history and examination has been very well described in the NICE drug allergy clinical guidelines, and we make no apologies for referring the reader to the assessment therein. ${ }^{3}$ The clinician is encouraged to

\section{Key points}

Drug allergy is common, but not as common as patients think

Accurate and appropriate documentation is essential for good management of drug allergy

Indications for specialist referral include suspected anaphylactic reaction and severe non-immediate cutaneous reaction

Skin prick testing, intradermal testing and drug provocation challenge are the main investigations used in the drug allergy clinic

Any drug can cause an allergic reaction, but most drug allergy referrals involve antibiotics, nonsteroidal anti-inflammatory drugs, general or local anaesthetics

KEYWORDS: Allergy, anaphylaxis, drug, intradermal testing, skin prick testing 


Box 1. Appropriate documentation in suspected
drug allergy.
$>$ Generic and proprietary name of suspected drug(s)
$>$ Route of administration
$>$ Date and time of reaction
$>$ Description of the reaction
$>$ Time interval or number of doses taken before reaction
$>$ The indication for the drug
$>$ Any previous reaction to suspected or related drugs
$>$ Other drugs being taken
$>$ Advice on which drugs or drug classes to avoid
Adapted from National Institute for Health and Care clinical guideline for drug
allergy.

utilise the boxes $1-3$ as described in the NICE guideline. This box system differentiates reactions based on timing, systemic involvement and severity. The distinction is made between immediate versus non-immediate; non-immediate being divided into systemic and non-systemic. Immediate reactions are more likely to be IgE-mediated, whereas non-immediate more likely to be cell-mediated in origin.

When assessing a patient with suspected drug allergy, it is always important to document appropriately, including the information summarised in Box 1. This information is also important to provide when referral is required to a specialist drug allergy clinic.

\section{Recommendations for referral to specialist services}

Recommendations with respect to referral to specialist services have been made both in the NICE clinical guideline on drug allergy and in the NICE clinical guideline on anaphylaxis. ${ }^{3,6}$ It has been recommended that people be referred to a specialist drug allergy service if they have had:

$>$ a suspected anaphylactic reaction

$>$ a severe non-immediate cutaneous reaction, for example drug reaction with eosinophilia and systemic symptoms (DRESS), Stevens-Johnson syndrome, toxic epidermal necrolysis.

There were further recommendations made with respect to specific drug classes.

> Non-steroidal anti-inflammatory drugs (NSAIDs) - refer to a specialist drug allergy service if the patient has had a suspected allergic reaction to an NSAID with symptoms such as anaphylaxis, severe angioedema or asthmatic reaction.

> Beta-lactam antibiotics - refer people with suspected allergy to beta-lactam antibiotics to a specialist drug allergy service if they:

$>$ need treatment for a disease or condition that can only be treated by a beta-lactam antibiotic or

$>$ are likely to need beta-lactam antibiotics frequently in the future (for example, people with recurrent bacterial infections or immune deficiency).

Consider referring people to a specialist drug allergy service if they are not able to take beta-lactam antibiotics and at least one other class of antibiotics because of suspected allergies.

> Local anaesthetics - refer people to a specialist drug allergy service if they need a procedure involving a local anaesthetic that they are unable to have because of suspected allergy to local anaesthetics.

> General anaesthesia - refer people to a specialist drug allergy service if they have had anaphylaxis or another suspected allergic reaction during or immediately after anaesthesia.

Referral of patients to a specialist drug allergy service can also be suggested if drug desensitisation could benefit the management of the patient. The most common drug desensitisation referral would be for aspirin. Desensitisation can also be important for other drugs, such as certain chemotherapeutic agents. Patients who complain of multiple drug allergies may also warrant specialist referral.

\section{To whom should referral be made?}

The NICE clinical guidelines describe referral to a specialist drug allergy service, but do not specify the specialty. This is because appropriate services may be run by allergists, immunologists or dermatologists depending on availability of local expertise and interest in the subject. The direction of the referral may also differ to some extent depending on whether the reaction was immediate/suspected IgE-mediated, or delayed/suspected cell-mediated. The international consensus position paper on drug allergy recommends the specific allergy work-up should be carried out 4-6 weeks after complete resolution of all clinical symptoms and signs of a suspected drug hypersensitivity reaction. ${ }^{7}$ However, in rare instances where a drug is needed more urgently, assessment can be done sooner.

\section{What can we do in the drug allergy clinic?}

There are a whole range of management options. Testing will be described below, but may not be indicated for all referred patients. Advice regarding drugs/drug classes to avoid, advice on alternative drugs/drug classes to use and strategies to minimise future risk can all be provided, as well as drug desensitisation in selected cases. Testing can involve serum allergen-specific IgE measurement, skin prick testing (SPT) and intradermal testing (IDT) for suspected or alternative drugs. Challenge or provocation testing can be undertaken for the index drug, or for alternative drugs.

Specific IgE testing is limited in that it is only routinely available for a small number of drugs or chemicals, such as penicillins, insulins, chlorhexidine, suxamethonium and pholcodine. In fact, there are very few drugs where the specific IgE tests are of sufficient diagnostic quality to merit routine clinical use. In addition, for certain drugs, where specific IgE testing is available, not all the antigenic determinants (epitopes) are represented in the test. Hence, false negative results may occur, as is well recognised with penicillin. ${ }^{8}$ False positive specific IgE results are also recognised. ${ }^{9}$ Therefore, NICE recommends that specific IgE testing is not used to diagnose drug allergy in a non-specialist setting. ${ }^{3}$

SPT is useful for the investigation of suspected IgE-mediated reactions, but will not be helpful for investigating suspected 
cell-mediated/delayed type hypersensitivity. There are recommended concentrations, which are used in routine testing. ${ }^{10}$ It is also important to be aware of false positive results, which can occur for certain drugs with intrinsic histamine releasing activity (eg atracurium, mivacurium, morphine).

IDT is more sensitive but less specific than SPT to the same concentration. Therefore, it is usually undertaken at much lower concentrations of the test $\operatorname{drug}(\mathrm{s})$. These tests are at higher risk of causing systemic allergic reactions. Although IDT are typically read at 15-20 minutes for immediate reactions, they can also be read at 48 hours to look for delayed type hypersensitivity. SPT and IDT are usually conducted by allergists and immunologists in specialist drug allergy clinics. Patch testing can also be used in the assessment of cell mediated/delayed type hypersensitivity, and is usually carried out by dermatologists with a special interest in this area.

There are a number of in vitro tests (other than specific IgE) that are not yet routinely available for the assessment of drug allergy. These include histamine-release assays, basophil activation test and lymphocyte proliferation test. These may be available on a research basis in specialist centres. These in vitro tests have recently been reviewed by the European Academy of Allergy and Clinical Immunology (EAACI). ${ }^{11}$

Challenge or provocation testing can be risky and is a labourintensive procedure. Therefore, it is only carried out with specific indications and by specialist allergy services with full monitoring and resuscitation facilities available. ${ }^{2}$ Testing can involve the index drug, or drug alternatives. It is considered only after all other investigations (if validated) have been completed, if the diagnosis is still doubtful and management would be affected by conducting the drug challenge.

\section{Specific drug classes}

\section{Beta-lactam antibiotics}

It is important to be aware of the structure of beta-lactam antibiotics as allergic sensitisation can occur to the beta-lactam ring, thiazolidine ring or the side chain. Penicillins are too small to act as full antigens; instead they act as haptens by binding to tissue or serum proteins. After administration, penicillins undergo a rapid degradation, which results in the formation of benzylpenicilloyl, the major antigenic determinant. The remaining part of the penicillin molecule degrades to a range of derivatives that form so-called 'minor determinants. ${ }^{12}$

We can investigate the evidence of sensitisation to major and minor penicillin determinants by SPT and IDT. However, cephalosporins undergo much quicker degradation, so at present it is possible to perform tests to the native molecule only. The correct diagnosis of beta-lactam allergy requires a detailed, accurate history, followed by skin testing, which is useful in type I/IgE-mediated (SPT and, if necessary, IDT) and type IV/delayed type reactions (late reading of IDT only). ${ }^{12}$ If testing is indicated, ideally there should not be a prolonged delay after the reaction has occurred because a long interval between the reaction and skin testing reduces the likelihood of a positive response. Skin testing with non-penicillin betalactams is less validated because cephalosporin degradation products are not fully identified. Therefore, the negative predictive value of skin testing remains uncertain.
If the skin testing is negative, a drug provocation test with the culprit beta-lactam antibiotic is required to exclude or determine the diagnosis. Protocols for challenge testing need to reflect the severity and the timing of the reaction. The oral route is preferable. If this initial challenge is negative, BSACI guidelines recommend the patient should continue the challenge at home for a further 3-5 days at the therapeutic dose. All patients should be provided with a written emergency plan and rescue medications after the challenge clinic. It is recognised that oral provocation testing can be falsely negative in 3-6\% of cases because of a lack of cofactors, such as underlying infection, painkillers, alcohol or exercise. ${ }^{12}$

The provocation test can also be useful in cases of mild cutaneous non-immediate reactions because delayed IDT reading and patch tests are of low sensitivity. However, the provocation test is contraindicated in case of severe nonimmediate cutaneous systemic reactions, eg toxic epidermal necrolysis, Stevens-Johnson syndrome. There are various protocols available and, in the UK, it is recommended that dependent on the severity of the original reaction, either a fraction of the dose or the full dose is given on day 1 , followed by a course of treatment 1 week later (in the absence of a delayed reaction). ${ }^{12}$

\section{General anaesthesia}

Neuromuscular blocking agents (NMBA) are the most common cause of an allergic reaction during general anaesthesia, followed by antibiotics, NSAIDs, dyes, colloids, latex and chlorhexidine. ${ }^{5,13}$ The aim of investigation is to identify the cause of reaction and recommend a range of drugs likely to be safe in the future.

Some of the features of peri-anaesthetic reaction may be due to non-allergic causes:

\section{$>$ technical difficulties with intubation \\ underlying medical conditions, eg sepsis, blood loss \\ $>$ pharmacology of the administered drugs.}

It is important to take serum tryptase level immediately after the onset of the reaction (as soon as the patient's clinical status is stabilised) and then at 1-2 hours. ${ }^{2,6,14}$ There are limitations to the interpretation of tryptase results. A high result, with subsequent reduction in tryptase over time, is indicative that anaphylaxis occurred. A normal result does make anaphylaxis unlikely, but does not rule this out completely. Of course, an elevated tryptase does not indicate the culprit for the reaction and it is important to obtain the anaesthetic chart, clinical notes and drug chart to guide the outpatient investigations. Reactions to NMBAs, antibiotics, latex, chlorhexidine, contrast media and dyes can be investigated by skin tests and, in some cases, by intravenous or oral challenge. The skin tests are not useful in cases of allergy to opioids and NSAIDs and, if indicated, a challenge with the suspected drug is the only way of confirming the cause of the reaction. There are no cases of reaction to inhaled anaesthetics. ${ }^{13}$

The timing of reaction is important. Anaphylaxis to intravenous induction agents (NMBA, intravenous anaesthetics), antibiotics, colloids and dyes (eg Patent Blue V) occur within minutes, whereas reactions to latex, NSAIDs and chlorhexidine may have slower onset. There is significant cross-reactivity between NMBAs; if NMBA allergy is suspected; 
all drugs in this class should be tested to identify a safe option for the future. All NMBAs indicated by positive skin tests should be avoided, as the provocation test is impractical. ${ }^{13} \mathrm{An}$ emerging, though rare, trigger for perioperative anaphylaxis is sugammadex, an anaesthetic reversal agent, which can cause reactions at the end of the procedure. ${ }^{15}$

After the investigations, a letter should be provided to the patient, GP and referring specialist with the identified cause of the reaction, as well as any cross-reacting drugs, and potential safe options for future procedures.

\section{Local anaesthetics}

Reassuringly, proven allergy to local anaesthetics is very rare. Bhole et al ${ }^{15}$ reviewed 23 case series involving 2,978 patients. Only 29 of these patients had true IgE-mediated allergy to local anaesthetic, giving a reported prevalence of local anaesthetic allergy of $0.97 \%$. Testing for allergy to local anaesthetics involves SPT, and in some centres IDT. The negative predictive value for SPT has been estimated at $97 \% .{ }^{16}$ Challenge doses are given by subcutaneous ${ }^{17}$ or, in some centres, intrabuccal routes. ${ }^{18}$

Reported reactions during procedures where local anaesthetics are given are most commonly due to IgE-mediated allergy to other co-existent allergens (eg antibiotics, latex, chlorhexidine) or reactions to excipients/preservatives, such as methylparabens. Other causes include non-allergic side effects, psychomotor reactions, inadvertent intravenous administration and pharmacological effects of adrenaline in compound preparations, eg lignocaine with adrenaline. ${ }^{15,18}$

\section{Aspirin/NSAIDs}

Hypersensitivity reactions to aspirin/NSAIDs represent a common clinical problem, and these patients will avoid all non-selective NSAIDs, including over-the-counter preparations. Most patients do not need specialist referral. Paracetamol can usually be tolerated in these patients, although around $10 \%$ of aspirin/NSAID-allergic patients will also react to paracetamol. ${ }^{19}$

NICE guidelines recommend that the introduction of a COX-2 inhibitor can be considered outside specialist care, using the lowest starting dose, with only a single dose on the first day for patients with a history of mild allergic reaction to NSAIDs, who need an anti-inflammatory. ${ }^{3}$ Patients who have had anaphylaxis, severe angioedema or asthmatic reaction to an NSAID should be referred to specialist allergy services for assessment if they require an anti-inflammatory drug. It is important to be aware that patients with asthma and nasal polyps may also have aspirin/NSAID-sensitivity (Samter's triad), unless they are known to have tolerated NSAIDs within the previous year.

\section{Angiotensin-converting enzyme inhibitors}

Angiotensin-converting enzyme inhibitors (ACEI) remain a common cause of angioedema presenting in accident and emergency departments and in referrals to the allergy clinic. Surprisingly, ACEI angioedema is still not always well recognised; this is because onset can be delayed for many years after taking the drug without problem, and the individual risk is actually very low $(0.1-0.7 \%)$. In our own service, the practice is to screen the drug history of all the isolated angioedema (ie without urticaria) referrals, and we continue to find many patients on
ACEIs. Our practice is to advise discontinuation of the ACEI, but that episodes may still occur for months thereafter. Patients with a diagnosis of ACEI angioedema do not usually require referral for specialist review, unless episodes continue to occur. If, for prognostic reasons, an angiotensin receptor blocker (ARB) would be indicated, this can be tried; there is a much lower risk of angioedema $\left(0.07 \%\right.$ in the Transcend trial).$^{20}$ If there is no prognostic indication for $\mathrm{ARB}$, then other alternatives can be considered instead. A 2015 study described the use of icatibant (a bradykinin B2-receptor antagonist) in acute ACEI angioedema to be more rapid in effect than standard treatment with antihistamine and glucocorticoid. ${ }^{21}$

\section{Other drugs}

Other drugs that commonly present for assessment to the allergy clinic include non-beta lactam antibiotics, insulin, heparin, opiates, radiocontrast media, plasma expanders (gelatin, dextran), povidone iodine and corticosteroids. ${ }^{2}$ Rarely, drug allergy can relate to excipients contained in drug preparations, ${ }^{22}$ although this should not be confused with intolerance, for example to lactose in the preparation.

\section{Drug desensitisation}

We would suggest referring patients to a drug allergy service if drug desensitisation is being considered as an important management option - such as when there are no satisfactory alternatives to the culprit drug. ${ }^{7}$ The most common indication in clinical practice would be for aspirin desensitisation in patients requiring aspirin thromboprophylaxis, although this can also be performed in selected patients as part of therapy in severe chronic polypoid rhinosinusitis.

Desensitisation can also be important for other drugs, such as antibiotics (under certain circumstances), ${ }^{23}$ platinumbased chemotherapeutic agents, taxenes and biologic agents/ monoclonal antibodies.

\section{Prevention of future reactions}

There are several aspects to the major goal of preventing future reactions. These include the following measures:

$>$ provide written information to patients and carers (patient education), and ask that this information be carried on their person

$>$ advise patient to join the Medicalert scheme where indicated, eg if there is a risk of intravenous use in emergency situations (penicillins, muscle relaxants, opiates) or for common overthe-counter drugs (NSAIDs) ${ }^{2}$

$>$ improve documentation of drug allergy status and new suspected drug allergy reactions, as described in NICE clinical guidelines ${ }^{3}$

> improve communication between healthcare professionals by providing drug allergy information in referral letters between general practice and hospital, and in hospital discharge summaries

> Use the Yellow Card Scheme for reporting ADRs.

Prescription of adrenaline autoinjectors is not usually required for allergic reactions as the mainstay of management is allergen avoidance to prevent future reactions. 


\section{Conflicts of interest}

MYK was a member of the NICE Quality Standards Advisory Committee for the 2015 drug allergy quality standard (QS97) and is the chair of the British Society for Allergy and Clinical Immunology's Clinical Immunology Subcommittee.

\section{References}

1 Edwards IR, Aronson JK. Adverse drug reactions: definitions, diagnosis and management. Lancet 2000;356:1255-9.

2 Mirakian R, Ewan PW, Durham SR et al. BSACI guidelines for the management of drug allergy. Clin Exp Allergy 2009;39:43-61.

3 National Institute for Health and Care Excellence. Drug allergy: diagnosis and management. NICE clinical guideline No 183. London: NICE, 2014.

4 National Institute for Health and Care Excellence. Drug allergy. NICE quality standard No 97. London: NICE, 2015.

5 Blanca M, Romano A, Torres MJ et al. Update on the evaluation of hypersensitivity reactions to betalactams. Allergy 2009;64:183-93.

6 National Institute for Health and Care Excellence. Anaphylaxis: assessment and referral after emergency treatment. NICE clinical guideline No 134. Manchester: NICE, 2011.

7 Demoly P, Adkinson NF, Brockow K et al. International consensus on drug allergy. Allergy 2014;69:420-37.

8 Fontaine C, Mayorga C, Bousquet PJ et al. Relevance of the determination of serum-specific IgE antibodies in the diagnosis of immediate beta-lactam allergy. Allergy 2007;62:47-52.

9 Johansson SG, Adédoyin J, van Hage M, Grönneberg R, Nopp A. False-positive penicillin immunoassay: an unnoticed common problem. J Allergy Clin Immunol 2013;132:235-7.

10 Brockow K, Garvey LH, Aberer W et al. Skin test concentrations for systemically administered drugs - an ENDA/EAACI Drug Allergy Interest Group position paper. Allergy 2013;68:702-12.

11 Mayorga C, Celik G, Rouzaire P et al. In vitro tests for drug hypersensitivity reactions: an ENDA/EAACI Drug Allergy Interest Group Position Paper. Allergy 2016:71;1103-34.

12 Mirakian R, Leech SC, Krishna MT et al. Management of allergy to penicillins and other beta-lactams. Clin Exp Allergy 2015;45:300-27.

13 Ewan PW, P Dugué, Mirakian R et al. BSACI guidelines for the investigation of suspected anaphylaxis during general anaesthesia. Clin Exp Allergy 2010;40:15-31.
14 Ebo DG, Fisher MM, Hagendorens MM, Bridts CH, Stevens WJ. Anaphylaxis during anaesthesia: diagnostic approach. Allergy 2007;62:471-87.

15 Ue KL, Kasternow B, Wagner A, Rutkowski R, Rutowski K. Sugammadex - an emerging trigger of intraoperative anaphylaxis. Clin Exp Allergy 2016; in press.

16 Bhole MV, Manson AL, Seneviratne SL, Misbah SA. IgE-mediated allergy to local anaesthetics: separating fact from perception: a UK perspective. Br J Anaesth 2012;108:903-11.

17 McClimon B, Rank M, Li J. The predictive value of skin testing in the diagnosis of local anesthetic allergy. Allergy Asthma Proc 2011;32:95-8.

18 Specjalski K, Kita-Milczarska K, Jassem E. The negative predictive value of typing safe local anesthetics. Int Arch Allergy Immunol 2013;162:86-8.

19 Rood JP. Adverse reaction to dental local anaesthetic injection'allergy' is not the cause. Br Dent J 2000;189:380-4.

20 Nettis E, Marcandrea M, Ferrannini A, Tursi A. Tolerability of nimesulide and paracetamol in patients with NSAID-induced urticaria/ angioedema. Immunopharmacol Immunotoxicol 2001;23:343-54.

21 Telmisartan Randomised Assessment Study in ACE iNtolerant subjects with cardiovascular Disease (TRANSCEND) Investigators, Yusuf S, Teo K et al. Effects of the angiotensin-receptor blocker telmisartan on cardiovascular events in high-risk patients intolerant to angiotensin-converting enzyme inhibitors: a randomised controlled trial. Lancet 2008;372:1174-83.

$22 \mathrm{Ba} \mathrm{M}$, Greve J, Stelter K et al. A randomized trial of icatibant in ACE-inhibitor-induced angioedema. N Engl J Med 2015;372:418-25.

23 Hegde VL, Venkatesh YP. Anaphylaxis to excipient mannitol: evidence for an immunoglobulin E-mediated mechanism. Clin Exp Allergy 2004;34:1602-9.

24 Demoly P, Messaad D, Sahla H et al. Six-hour trimethoprimsulfamethoxazole-graded challenge in HIV-infected patients. J Allergy Clin Immunol 1998;102:875-6.

Address for correspondence: Dr M Y Karim, Clinical Immunology \& Allergy, Frimley Park Hospital, Portsmouth Road, Frimley, Surrey GU16 7UJ, UK.

Email: yousuf.karim@nhs.net 